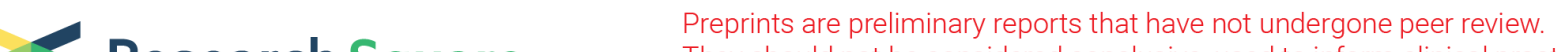

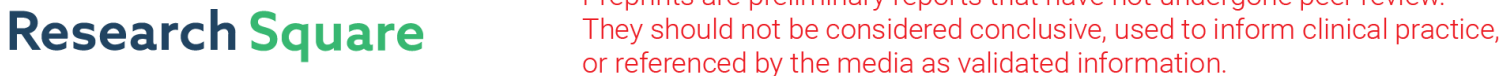

\section{Plaque Regrowth Dynamics with Use of an Oscillating-Rotating Electric Toothbrush Measured by Digital Plaque Image Analysis: A Randomized Controlled Trial}

Priscila Ferrari Peron ( $\square$ priscila.peron@unimedizin-mainz.de)

Department of Orthodontics, University Medical Center of the Johannes Gutenberg-University, Mainz Ralf Adam

Procter \& Gamble Service GmbH, German Innovation Center Julie Grender

The Procter \& Gamble Company, Mason Business Center

Uta Mesples

Procter \& Gamble Service GmbH, German Innovation Center

Phyllis Hoke

The Procter \& Gamble Company, Mason Business Center

\section{Mike Rubush}

The Procter \& Gamble Company, Mason Business Center

\section{Mary Kay Anastasia}

The Procter \& Gamble Company, Mason Business Center

\section{Christina Erbe}

Department of Orthodontics, University Medical Center of the Johannes Gutenberg-University, Mainz

\section{Research Article}

Keywords: electric toothbrush, dental plaque, oscillating-rotating, digital imaging

Posted Date: February 7th, 2022

DOI: https://doi.org/10.21203/rs.3.rs-1317441/v1

License: (c) (1) This work is licensed under a Creative Commons Attribution 4.0 International License. Read Full License 


\section{Abstract}

This study aimed to compare dental plaque regrowth dynamics across 8 days between an oscillatingrotating (O-R) electric toothbrush and a manual toothbrush using Digital Plaque Imaging Analysis (DPIA). A randomized crossover trial involving adult participants with $\geq 10 \%$ plaque coverage on anterior teeth was conducted. Thirty-four subjects were randomized to one of 4 treatment sequences, each having four 8-day treatment periods. Subjects brushed twice daily with the assigned toothbrush. Morning and afternoon plaque were evaluated on days 1,3 , and 8 . The primary variable was afternoon percent plaque coverage, evaluated across 8 days.

The O-R toothbrush produced lower mean plaque levels versus the manual toothbrush for all endpoints over 8 days ( $P \leq 0.001)$. The benefit for the O-R toothbrush increased over time, demonstrating $16.4 \%$ and $28.1 \%$ lower afternoon plaque levels on Days 1 and 8, respectively compared to the manual brush. Trends were similar for morning plaque. Repeated measures analyses showed a $21.2 \%$ reduction in overall afternoon plaque and a $23.5 \%$ reduction in overall morning plaque for the $0-\mathrm{R}$ brush versus the manual brush $(P<0.001)$. The $0-\mathrm{R}$ toothbrush resulted in less overnight and afternoon plaque regrowth versus the manual toothbrush at all time points, and the benefit increased over 8 days.

\section{Background}

Gingivitis is a widespread oral disease typically resulting from bacterial plaque ${ }^{1}$. This condition affects a majority of the global population and is commonly associated with lack of effective daily oral hygiene $e^{2,3}$. New research shows that as early as 24 to 72 hours after oral hygiene is stopped, before symptoms of gingival bleeding and inflammation typically appear, the oral profile of bacterial species, bacterial metabolites, and host cytokines begins a characteristic shift toward a state of gingivitis ${ }^{4}$. This rapid shift in the oral microbiome underscores the importance of early intervention.

The key to preventing gingivitis and its sequelae is plaque control ${ }^{1,5}$, and the cornerstone of plaque control is mechanical removal by patients themselves via at-home toothbrushing ${ }^{5-7}$. In multiple studies, electric toothbrushes have been shown to be superior to manual toothbrushes at removing plaque and reducing gingivitis ${ }^{6,8-11}$. Among electric brushes, those with oscillating-rotating (O-R) movement have demonstrated efficacy advantages over those with side-to-side (sonic) movement ${ }^{6,11-13}$.

Historically, plaque has been evaluated by an examiner using a subjective index to assign numerical values, representing the amount of observed plaque on various regions of the tooth. These methods emphasize plaque assessment at the gingival margin ${ }^{14-16}$ since plaque removal is notoriously problematic in this region, making them well-suited for studies of plaque removal ${ }^{17,18}$. In contrast, digital plaque imaging analysis (DPIA) is an objective and sensitive way to evaluate total plaque ${ }^{19}$. In this method, fluorescein-disclosed plaque is illuminated by ultraviolet (UV) light and a digital image is captured. Tooth plaque, identified in the image by a unique color value, can be precisely quantitated by summing the number of pixels in that color category and then calculating the percentage of the tooth 
covered with plaque. Because the DPIA method is equally sensitive over the entire tooth surface, it is particularly useful for studies assessing plaque regrowth ${ }^{20-23}$, which has been observed to begin in the interproximal areas and extend to facial tooth surfaces ${ }^{24}$.

Recently, an O-R toothbrush with micro-vibrations (Oral-B® iO) $)^{25,26}$ has demonstrated greater plaque removal and gingivitis reduction relative to that of both manual and sonic toothbrushes. This level of performance has been observed after a single brushing and throughout studies lasting 8, 12, and 24 weeks ${ }^{17,27-29}$. The aim of this study was to understand the increasing effect with brushing of the O-R brush on plaque regrowth dynamics throughout an 8-day period of use, compared with the effect of a reference manual brush.

\section{Methods}

The protocol was reviewed and approved by the ethics committee of the State Medical Board of Rhineland-Palatinate, Mainz, Germany (Ref: 2020-14928) and registered as ISRCTN:

https://www.isrctn.com/ISRCTN28649560. The study was conducted in accordance with applicable the International Conference on Harmonization's Good Clinical Practice guidelines and the European Union's Commission Directive 2005/28/EC. All subjects provided written, informed consent.

\section{Study design and settings}

This was a randomized, single-center, 2-treatment, 4-period crossover trial conducted at the University Medical Center of the Johannes Gutenberg-University. The objective of this study was to compare an O-R electric rechargeable toothbrush and a reference manual toothbrush with respect to adult users' rate of overnight and daytime plaque regrowth during an 8-day period, as assessed by DPIA.

\section{Sample size calculation}

Based on previous data and using a 2-sided test with $a=.05$, it was determined that 40 subjects provided the study $80 \%$ power to detect a significant mean treatment difference of 1.44 for afternoon DPIA plaque over 5 days, with an estimated variability of 1.146. Due to COVID-related circumstances, we were able to recruit 34 subjects meeting inclusion criteria.

\section{Patient recruitment and follow-up}

Subjects were recruited by the University Medical Center of the Johannes Gutenberg-University from the Center's patient population between July and August 2020. Subjects were asked to refrain from toothbrushing for 24 hours before the screening appointment, at which time they provided written, informed consent, personal medical history, and demographic information. Subjects meeting inclusion criteria received a comprehensive examination of hard and soft oral tissues, and then disclosed plaque according to the fluorescein plaque-disclosing procedure after which time a pre-brushing DPIA image was taken of the anterior teeth. 


\section{Eligibility criteria}

Eligible subjects were adults in good general health with an estimated plaque coverage of at least $10 \%$ on the buccal surfaces of the anterior teeth, as assessed by DPIA approximately 24 hours after brushing. Eligible subjects had at least 10 natural anterior teeth with scorable facial surfaces (excluding crowns, excessive facial restorations, or severe staining from tetracycline, fluorosis, or hypocalcification). Exclusion criteria included participation in any other oral care study, use of non-study oral hygiene products, elective dentistry, caries lesions that required restoration, calculus on facial tooth surfaces, active treatment for periodontitis, fixed facial or lingual orthodontic appliances, use of an antibiotic or chlorhexidine mouth rinse within 2 weeks prior to screening visit, pregnancy, lactation, hypersensitivity to dyes, or any disease or condition that could be expected to interfere with study procedures. Subjects agreed to refrain from all oral hygiene procedures for 24 hours prior to each morning visit and between each morning on-site brushing and the afternoon imaging visit. Subjects also agreed to abstain from eating, drinking, chewing gum, and using tobacco for 2 hours prior to each morning appointment and for 1 hour prior to each afternoon appointment. Small sips of water were permitted up to 1 hour before each morning appointment and the use of dental floss was permitted between treatment periods.

\section{Safety}

Serious adverse events (AEs), oral-related AEs, and whole-body AEs that could possibly be related to the study products were recorded upon self-report.

\section{Processes, interventions, and comparisons}

Treatments were (A) an experimental electric toothbrush (Oral-B iO OP020-PS2.0) with accompanying brush head (Oral-B Ultimate Clean OR015-PS2.0) [Procter \& Gamble, Cincinnati, OH, United States] and (B) a control manual toothbrush (Oral-B® Indicator 35 soft) [Procter \& Gamble]. All products were used with a standard sodium fluoride dentifrice (Blend-a-Med Classic, $1450 \mathrm{ppm}$ fluoride as sodium fluoride) [Procter \& Gamble].

At the screening visit, enrolled subjects began an initial acclimation phase of 2 to 3 days to acclimate to the electric toothbrush. Subjects were provided with kit boxes containing instructions, the electric toothbrush handle and brush head, and one tube of toothpaste. After receiving product use instructions, subjects brushed on site and were then instructed to use the electric toothbrush for 2 minutes twice a day with the provided toothpaste during the acclimation phase. This was followed by a second phase, following a similar process, to acclimate subjects to the manual toothbrush. Subjects were instructed to use the manual toothbrush in their customary manner twice a day with the provided toothpaste. The manual toothbrush was also used as the washout product between treatment periods to prevent potential carry-over.

After both acclimation phases, 34 subjects were each randomly assigned to 1 of the following 4 treatment sequences, each consisting of 4 treatment periods: $A B B A, B A A B, A A B B$, or $B B A A$, with $A$ and $B$ 
representing the electric and manual toothbrush, respectively. Treatment periods lasted 8 days, including study visits on days 1,3 , and 8 .

On day 1 of each period, continuance criteria were assessed, acclimation products were collected, and treatment kit boxes (consisting of instructions, the assigned toothbrush, and one tube of toothpaste) were distributed for use throughout the period.

On the morning of each visit (days 1, 3, and 8), each subject received an oral examination followed by plaque disclosure and pre-brushing DPIA plaque measurement of the anterior teeth. Brushing instructions were then reviewed, and subjects performed a supervised brushing (deemed their first brushing of the day) with their assigned brush. Subjects returned to the study site approximately 5 hours later for an afternoon assessment. At that time, plaque was again disclosed and a post-brushing DPIA plaque measurement was conducted of the anterior teeth. After the afternoon assessment of day 8 for treatment periods 1,2 , and 3 , treatment products were collected, washout product kits (instructions, a manual toothbrush, and one tube of toothpaste) were distributed, and subjects were reminded to use the washout products twice daily during the 6-day washout period.

Continuance criteria were reviewed at each visit. Study procedures were repeated until all subjects had completed 4 treatment periods according to their randomly assigned treatment sequence.

\section{DPIA imaging procedure}

Prior to each imaging, subjects disclosed plaque by rinsing for 10 seconds with $25 \mathrm{ml}$ phosphate buffer, then rinsing for 1 minute with $5.0 \mathrm{ml}$ of $1240 \mathrm{ppm}$ fluorescein in phosphate buffer, and finally rinsing 3 times for 10 seconds each with $25 \mathrm{ml}$ phosphate buffer. The DPIA imaging system consisted of a highresolution digital camera (Canon Corporation), $55 \mathrm{~mm}$ macro lens, and UV barrier filter, lit on each side by a compact flash unit with a long wave UV ( $365 \mathrm{~nm}$ peak) blue filter. A trained and validated operator captured images using validated image capture software. The system was initially calibrated to color standards values and then standardized daily with a calibrated color chart to ensure performance within calibration limits. The color chart was imaged every 30 minutes during use to ensure continued, standardized performance. Digital images were captured according to the procedure developed by Sagel et al. ${ }^{19}$ as described by Erbe et $\mathrm{al}^{21}$.

\section{Primary and secondary outcome measures}

The primary variable was afternoon plaque DPIA score, evaluated across 8 days. Secondary outcome measures included the morning pre-brushing DPIA measure. The null hypothesis was that the 2 toothbrush types would show no difference with respect to average DPIA after adjusting for baseline DPIA score. The alternative hypothesis was that the toothbrushes would show a difference with respect to average DPIA after adjusting for baseline DPIA score.

\section{Statistical analysis}


Summary statistics were calculated for the demographic variables and AEs were collected for each treatment group. Treatment effects were analyzed with 2-sided statistical tests and an $a=.05$ significance level. There were no adjustments for multiple comparisons.

\section{Morning and Afternoon}

For morning DPIA plaque coverage (days 3 and 8 ) and afternoon DPIA plaque coverage (on days 1,3 , and 8), an analysis of covariance (ANCOVA) for a crossover design was used. Assessment was conducted separately for each day, with the day 1 pre-brushing DPIA measurement used as the covariate. The model also included terms for subject as a random effect and treatment, period, and covariate by treatment interaction as fixed effects. Carryover effects were not significant at the $10 \%$ level, and thus were not included in the final model.

\section{Weekly plaque reduction}

An ANCOVA for a crossover design was used to assess the weekly reduction in pre-brushing plaque (day 1 pre-brushing minus day 8 pre-brushing DPIA measurements) with day 1 pre-brushing DPIA measurement as the covariate. The model also included terms for subject as a random effect and treatment, period, and covariate by treatment interaction as fixed effects. Carryover effects were not significant at the $10 \%$ level, and thus were not included in the final model.

\section{Repeated measures}

A repeated measures analysis across days was used to assess overall trends in morning pre-brushing (days 3 and 8) and afternoon post-brushing (days 1, 3, and 8) DPIA measurements. A repeated measures ANCOVA for a crossover design was used with the day 1 pre-brushing plaque as the covariate. The model also included terms for subject and subject by period interaction as random effects, and treatment, period, day, and baseline by treatment interaction as fixed effects. Carryover effects were not significant at the $10 \%$ level, and thus were not included in the final model.

\section{Results}

\section{Study population}

59 subjects were screened; 34 met all entrance criteria and were randomized to 1 of 4 treatment sequences. All 34 subjects ( $53 \%$ female) with a mean age of 37.9 years completed the study.

\section{Efficacy Data}

\section{Afternoon 5-Hour Post-Brushing Plaque Levels}

The O-R brush had statistically significantly $(p \leq 0.001)$ lower mean plaque levels compared to the manual control brush for the afternoon 5-hour post-brushing DPIA measures on all days, with $16.4 \%, 19.1 \%$ and 
$28.1 \%$ lower plaque levels on Days 1, 3 and 8 , respectively. The post-brushing plaque analysis across all visits showed the $0-R$ brush with a $21.2 \%$ lower plaque score compared to the manual brush $(p<0.001)$. See Table 1.

Table 1

Afternoon (5-hours post-brushing) DPIA results by day and across days. The percent treatment difference is the treatment mean difference divided by the manual brush adjusted mean.

\begin{tabular}{|lllll|}
\hline & $\begin{array}{l}\text { Adjusted } \\
\text { Mean (SE) }\end{array}$ & $\begin{array}{l}\text { Treatment } \\
\text { Difference (SE) }\end{array}$ & $\begin{array}{l}\text { 2-sided p- } \\
\text { value }\end{array}$ & $\begin{array}{l}\text { \% treatment } \\
\text { difference }\end{array}$ \\
\hline Day 1 & $9.00(0.670)$ & $1.47(0.449)$ & 0.001 & $16.4 \%$ \\
\hline Manual & $7.53(0.669)$ & & & \\
\hline O-R & & & & \\
\hline Day 3 & $8.43(0.591)$ & $1.61(0.484)$ & 0.001 & $19.1 \%$ \\
\hline O-R & $6.82(0.593)$ & & & \\
\hline Day 8 & & & & \\
\hline Manual & $9.17(0.609)$ & $2.58(0.498)$ & & \\
\hline O-R & $6.60(0.599)$ & & & \\
\hline $\begin{array}{l}\text { Repeated Measures } \\
\text { (across days) }\end{array}$ & & & & \\
\hline Manual & $8.86(0.573)$ & $1.87(0.379)$ & & \\
\hline O-R & $6.98(0.572)$ & & & \\
\hline
\end{tabular}

\section{Morning Plaque Levels}

There was no significant difference between treatments at baseline (Day 1) for morning pre-brushing plaque, with the $0-\mathrm{R}$ brush having $16.6 \%$ plaque coverage compared to $16.4 \%$ for the control manual brush $(p=0.890)$.

The O-R brush had statistically significantly $(p \leq 0.001)$ lower mean plaque levels compared to the manual control brush for morning pre-brushing plaque scores on Day 3 by $18.8 \%$ and Day 8 by $27.7 \%$.

Additionally, the 0-R brush showed $23.5 \%$ less morning pre-brushing plaque across all days compared to the manual brush $(p<0.001)$. See Table 2 . 
Table 2

Morning pre-brushing DPIA results by day and across days. The percent treatment difference is the treatment mean difference divided by the manual brush adjusted mean.

\begin{tabular}{|lllll|}
\hline & $\begin{array}{l}\text { Adjusted } \\
\text { Mean (SE) }\end{array}$ & $\begin{array}{l}\text { Treatment } \\
\text { Difference (SE) }\end{array}$ & $\begin{array}{l}\text { 2-sided p- } \\
\text { value }\end{array}$ & $\begin{array}{l}\text { \% treatment } \\
\text { difference }\end{array}$ \\
\hline Day 3 & & & & \\
\hline Manual & $15.57(0.925)$ & $2.92(0.858)$ & $<0.001$ & $18.8 \%$ \\
\hline O-R & $12.65(0.928)$ & & & \\
\hline Day 8 & & & & \\
\hline Manual & $16.54(0.790)$ & $4.57(1.104)$ & & \\
\hline O-R & $11.97(0.772)$ & & & \\
\hline $\begin{array}{l}\text { Repeated Measures } \\
\text { (across days) }\end{array}$ & & & & \\
\hline Manual & $16.09(0.735)$ & $3.78(0.823)$ & $<0.001$ & $23.5 \%$ \\
\hline O-R & $12.31(0.732)$ & & & \\
\hline
\end{tabular}

The consistently lower mean plaque levels for the O-R toothbrush compared to the manual toothbrush at all morning pre-brushing and afternoon 5-hour post-brushing visits can be seen in Figure 1.

\section{Safety Data}

There were four mild Adverse Events reported in the study with use of the manual brush. Three were classified as not related to product use and one was doubtful.

\section{Discussion}

In this first report of plaque regrowth dynamics for the novel O-R toothbrush, there was approximately $28 \%$ less overnight and daytime plaque regrowth with use of the O-R toothbrush compared to the control manual brush after 8 days of use. In addition, significantly less plaque was observed as early as the afternoon of day 1 , after a single brushing ( $16.4 \%$ less plaque among $0-\mathrm{R}$ brush users relative to manual brush users), and the plaque reduction benefit for the O-R brush increased over the course of a week (See Figure 2). These results confirm the plaque reduction advantage of the $0-\mathrm{R}$ brush relative to that of a manual brush after a single brushing, which has been demonstrated in previous studies ${ }^{27,29}$, and help to explain the sustainable long-term superiority of the $0-\mathrm{R}$ brush for both plaque and gingivitis reductions when compared to manual and sonic brushes in studies out to six months ${ }^{17,27-29}$. We hypothesize the 0$\mathrm{R}$ brush reduces the bacterial load more than the manual brush and thereby delays growth and reconstitution of the bacterial biofilm between brushing events. Over time, the greater plaque removal provided by the O-R brush versus the manual control brush creates an increasingly growing difference. A 
recent study evaluating the O-R brush as a part of a regimen with an antibacterial dentifrice and rinse indicates the use of chemotherapeutics in combination with the mechanical action of the O-R brush routine increases the plaque benefit further ${ }^{30}$.

The current study also demonstrates that plaque coverage quantification provided by DPIA may offer greater sensitivity compared to established plaque indices for plaque regrowth evaluations. Earlier research on plaque regrowth has shown that at low plaque levels, daily post-brushing residual plaque accumulated but remained undetectable by plaque index (PI) analysis ${ }^{24}$. The authors found that planimetric analysis from photographs (a precursor to modern DPIA) showed more pronounced differentiation between the study groups than was distinguished by mean plaque index (MPI) scores, indicating room for improvement over MPI as a means of quantifying plaque coverage. This observation was particularly pronounced at a PI score below 0.4 and MPI below $20 \mathrm{~cm}^{2}(\sim 10 \%$ plaque coverage) where there was little association between indices. This is similar to the plaque levels in this trial. While DPIA is unique compared to traditional examiner-based plaque assessments because it only includes anterior teeth, research has shown evaluation of plaque on anterior teeth is a reliable estimate of whole mouth plaque (intraclass correlation $=0.743)^{31}$.

In future studies of this novel O-R toothbrush, DPIA will be a useful tool for understanding plaque regrowth dynamics and how they influence gingivitis onset and progression. It would be particularly interesting to focus on subjects with difficult anatomical features, such as misaligned anterior teeth, since the O-R brush has shown disproportionate plaque removal advantages in areas that are usually difficult to clean ${ }^{17,27}$. Presently, DPIA has shown that the Oral-B $\mathrm{iO}{ }^{\circledR}$ toothbrush removes more plaque and keeps plaque levels consistently lower overnight and throughout the day when compared to a manual toothbrush, and importantly the between-treatment difference increased over time for both the overnight plaque and afternoon plaque regrowth. Given the speed with which the oral microenvironment develops characteristics of gingivitis ${ }^{4}$, this type of sustainable plaque control is an important step toward reducing the risk of periodontal disease.

\section{Conclusions}

In this study, a novel O-R brush prevented overnight and daytime plaque regrowth better than a manual brush. Superiority in relative plaque reduction was observed at first use and persisted throughout a week of use. By removing more plaque and keeping plaque levels consistently lower throughout the day and night, this novel O-R brush offers more effective plaque control at each brushing compared to a regular manual toothbrush, which is consistent with the sustained long-term plaque and gingivitis reductions demonstrated by the O-R brush versus controls in multiple clinical trials ${ }^{17,27-29}$. This study also demonstrated the utility of DPIA as an elegant in vivo method to evaluate cleaning potential of toothbrush technologies over time, which will inform future studies of the relationship between plaque regrowth dynamics and gingivitis. 


\section{Declarations}

Acknowledgements

The authors thank Manuela Dashtiary, study nurse, for her excellent support with study execution and Marisa DeNoble Loeffler, MS, for medical writing assistance in the preparation of the manuscript.

\section{Author contributions}

C.E. was the principal investigator and participated in study design and execution. R.A. and J.G. participated in study design and in the analysis and interpretation of data. M.K.A. contributed to study execution and data analysis. P.F.P., U.M., P.H., and M.R. also contributed to the execution of the study. All authors approved the submitted manuscript and agree to be personally accountable for the work.

\section{Additional Information}

R.A., J.G., U.M., P.H., M.R., and M.K.A. are employees of Procter \&Gamble, the manufacturer of the O-R toothbrush evaluated in the study. P.F.P. and C.E. declare no conflicts of interest.

\section{Funding}

The study was funded by The Procter \& Gamble Company. Medical writing assistance was funded by The Procter \& Gamble Company.

\section{References}

1. Löe, H., Theilade, E. \& Jensen, S. Experimental gingivitis in man. J. Periodont. 36, 177-187 (1965).

2. Li, Y. et al. Prevalence and severity of gingivitis in american adults. Am. J. Dent. 23, 9-13 (2010).

3. Gasner, N. S. \& Schure, R. S. Periodontal Disease. PMID: 32119477. (StatPearls, 2021).

4. Huang, S. et al. Longitudinal multi-omics and microbiome meta-analysis identify an asymptomatic gingival state that links gingivitis, periodontitis, and aging. mBio12, e03281-20 (2021).

5. Ower, P. The role of self-administered plaque control in the management of periodontal diseases: I. A review of the evidence. Dent. Update. 30, 60-64,66, 68 (2003).

6. Van der Weijden, F. \& Slot, D. Efficacy of homecare regimens for mechanical plaque removal in managing gingivitis a meta review. J. Clin. Periodont.42 Suppl 16, S77-91 (2015).

7. Ower, P. et al. The role of self-administered plaque control in the management of periodontal diseases: 2. Motivation, techniques and assessment. Dent. Update. 30, 110-6 (2003).

8. Yaacob, M. Powered versus manual toothbrushing for oral health. Cochrane Database Syst. Rev.6, CD002281 (2014).

9. Pitchika, V. et al. Long-term impact of powered toothbrush on oral health: 11-year cohort study. J. Clin. Periodontol. 46, 713-22 (2019). 
10. Elkerbout, T., Slot, D., Rosema, N. \& Van der Weijden, G. How effective is a powered toothbrush as compared to a manual toothbrush? A systematic review and meta-analysis of single brushing exercises. Int. J. Dent. Hyg.18, 17-26 (2020).

11. Grender, J., Adam, R. \& Zou, Y. The effects of oscillating-rotating electric toothbrushes on plaque and gingival health: a meta-analysis. Am. J. Dent.33, 3-11 (2020).

12. Deacon, S. A. et al. Different powered toothbrushes for plaque control and gingival health. Cochrane Database Syst. Rev.12, CD004971 (2010).

13. Clark-Perry, D. \& Levin, L. Systematic review and meta-analysis of randomized controlled studies comparing oscillating-rotating and other powered toothbrushes. J. Am. Dent. Assoc.151, 265-275.e6. (2020).

14. Quigley, G. A. \& Hein, J. W. Comparative cleansing efficiency of manual and power brushing. J. Am. Dent. Assoc.65, 26-29 (1962).

15. Turesky, S., Gilmore, N. D. \& Glickman, I. Reduced plaque formation by the chloromethyl analogue of victamine c. J. Periodontol. 41, 41-43 (1970).

16. Rustogiy, K. N. et al. Refinement of the Modified Navy Plaque Index to increase plaque scoring efficiency in gumline and interproximal tooth areas. J. Clin. Dent.3 Suppl C, C9-12 (1992).

17. Goyal, C. R., Adam, R., Timm, H., Grender, J. \& Qaqish, J. A 6-month randomized controlled trial evaluating a novel smart-connected oscillating-rotating toothbrush versus a smart-connected sonic toothbrush for the reduction of plaque and gingivitis. Am. J. Dent. 34, 54-60 (2021).

18. Sharma, N. C., Qaqish, J., Walters, P. A., Grender, J. \& Biesbrock, A. R. A clinical evaluation of the plaque removal efficacy of five manual toothbrushes. J. Clin. Dent.21, 8-12 (2010).

19. Sagel, P. A., Lapujade, P. G., Miller, J. M. \& Sunberg, R. J. Objective quantification of plaque using digital image analysis. Monogr. Oral Sci. 17, 130-43 (2000).

20. Bellamy, P. G. et al. Randomized in vivo trial evaluating plaque inhibition benefits of an advanced stannous-containing sodium fluoride dentifrice used in conjunction with power brush technology. Int. J. Dent. Hyg. 12, 89-95 (2014).

21. Erbe, C. et al. Efficacy of 3 toothbrush treatments on plaque removal in orthodontic patients assessed with digital plaque imaging: a randomized controlled trial. Am. J. Orthod. Dentofacial Orthop.143, 760-766 (2013).

22. White, D. J. et al. A 24-hour dental plaque prevention study with a stannous fluoride dentifrice containing hexametaphosphate. J. Contemp. Dent. Pract.7, 1-11 (2006).

23. White, D. J. Effect of a stannous fluoride dentifrice on plaque formation and removal: a digital plaque imaging study. J. Clin. Dent.18, 21-4. (2007).

24. Lang, N. P., Cumming, B. R. \& Löe, H. Toothbrushing frequency as it relates to plaque development and gingival health. J. Periodontol. 44, 396-405 (1973).

25. Adam, R. Introducing the Oral-B iO electric toothbrush: next generation oscillating-rotating technology. Int. Dent. J. 70 Suppl 1, S1-6 (2020). 
26. Goldschmidtboeing, F. et al. Bristle motion, forces, and related vertical translation for a novel electric toothbrush design. J. Mech. Eng.66, 505-12 (2020).

27. Grender, J., Goyal, C., Qaqish, J. \& Adam, R. An 8-week randomized controlled trial comparing the effect of a novel oscillating-rotating toothbrush versus a manual toothbrush on plaque and gingivitis. Int. Dent. J.70 Suppl 1, S7-15 (2020).

28. Adam, R., Goyal, C., Qaqish, J. \& Grender, J. Evaluation of an oscillating-rotating toothbrush with micro-vibrations versus a sonic toothbrush for the reduction of plaque and gingivitis: Results from a randomized controlled trial. Int. Dent. J.70 Suppl 1, S16-21 (2020).

29. Adam, R., Erb, J. \& Grender, J. Randomized controlled trial assessing plaque removal of an oscillating-rotating electric toothbrush with micro-vibrations. Int. Dent. J. 70 Suppl 1, S22-7 (2020).

30. Adam, R., Grender, J., Timm, H., Qaqish, J. \& Goyal, C. R. Anti-gingivitis and anti-plaque efficacy of an oral hygiene system: results from a 12-week randomized controlled trial. Compend. Contin. Educ. Dent.42, E1-4 (2021).

31. Dunavent, J. M., Barker, M. L., Gerlach, R. W., Singh, M. \& Papas, A. S. Partial versus whole mouth grading of disclosed plaque. J. Dent. Res. 87(Spec Iss B), Abstract 1280 (2008).

\section{Figures}

\section{DPIA Plaque Coverage}

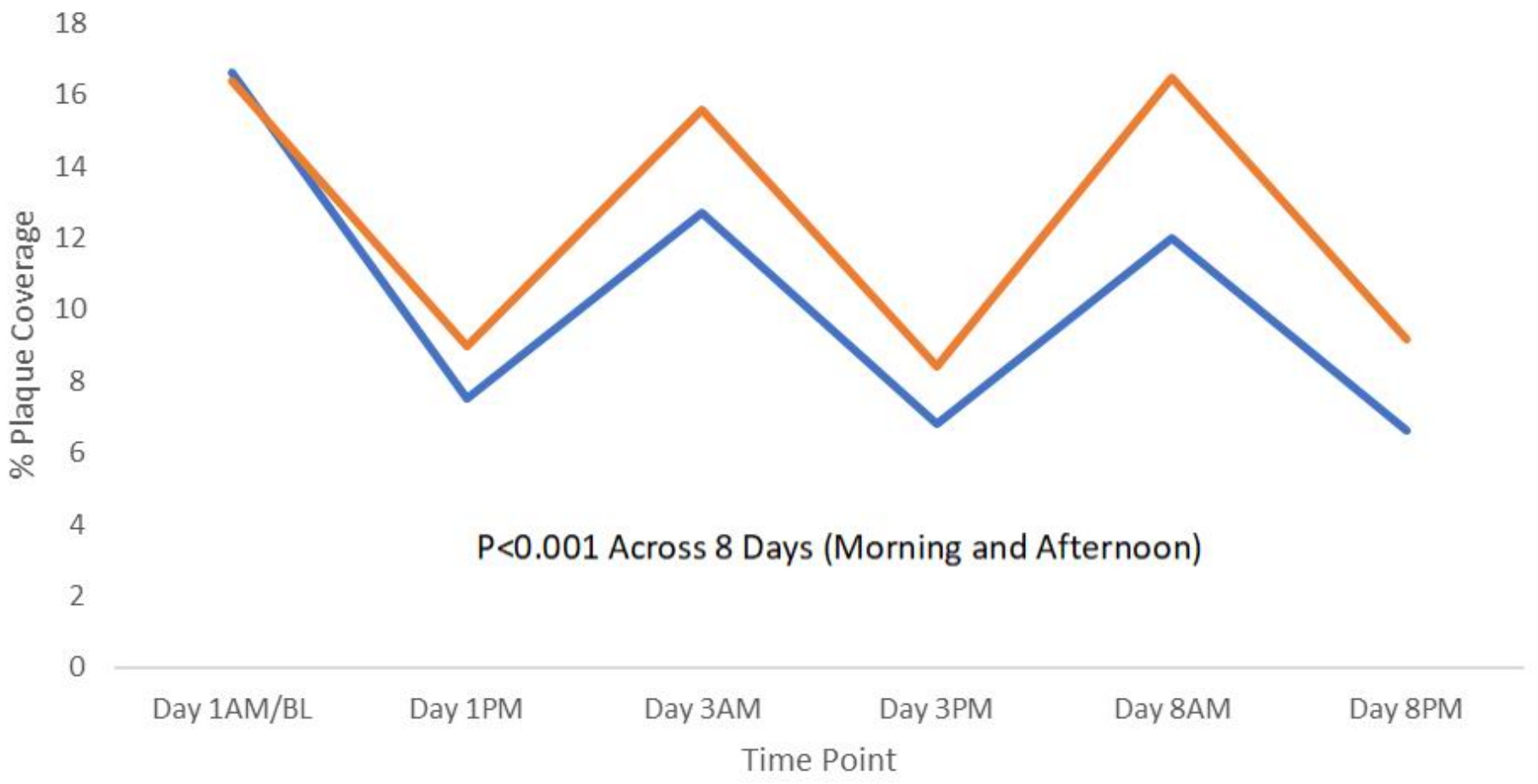


Percent plaque coverage for all visits.

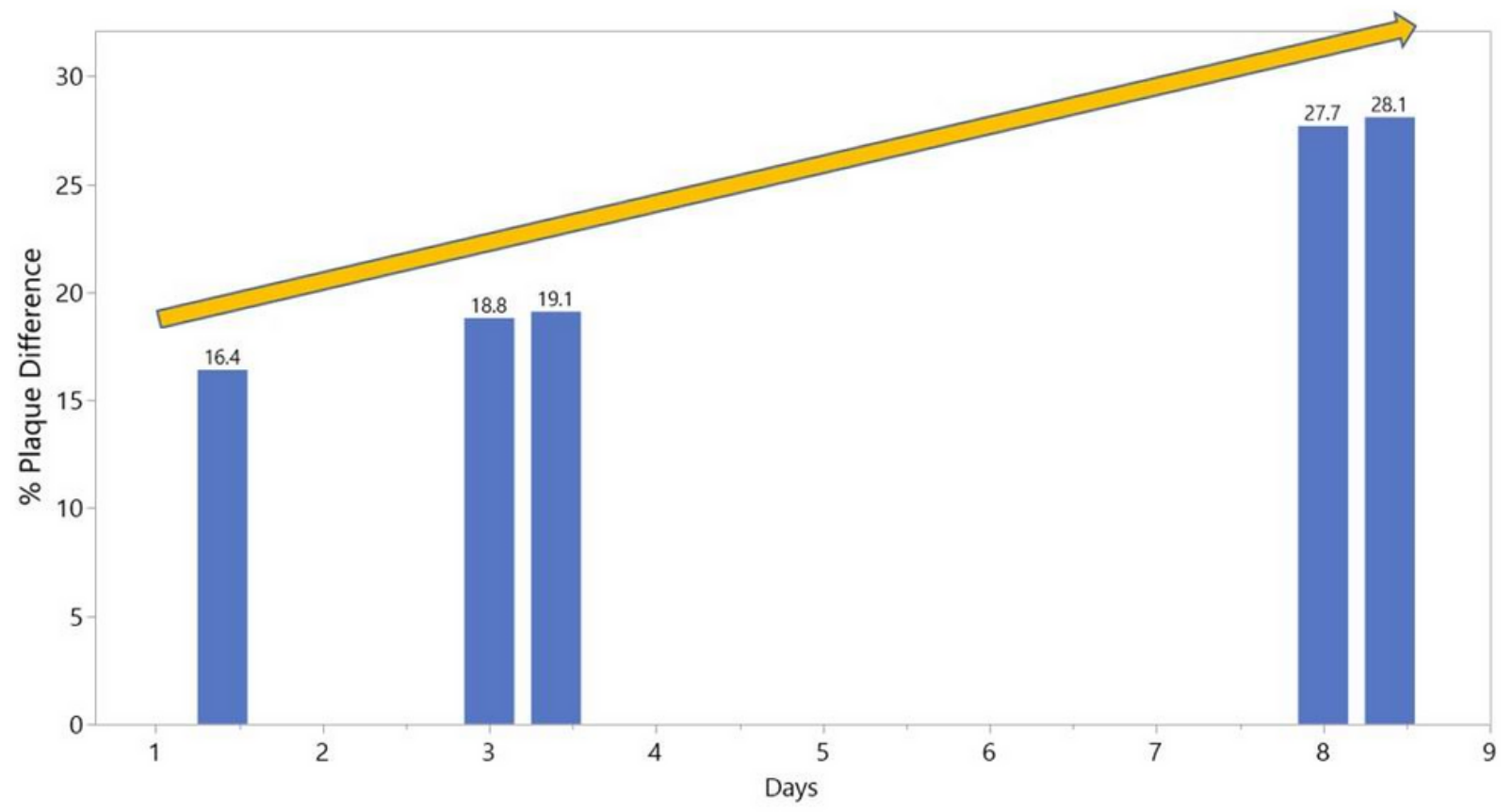

Figure 2

Percent plaque difference between toothbrushes, favoring the O-R toothbrush, over the treatment period. Bars represent both afternoon and morning plaque. 